\title{
Vibrio isolates from cases of acute diarrhea and their antimicrobial susceptibility pattern in a tertiary care hospital
}

\author{
Ekta Mala*, Aroma Oberoi, Vipin Sam Alexander \\ Department of microbiology, Christian medical college and hospital, Ludhiana \\ *Corresponding authorE-mail: ritumicro86@gmail.com
}

Copyright $\odot 2014$ Ekta Mala et al. This is an open access article distributed under the Creative Commons Attribution License, which permits unrestricted use, distribution, and reproduction in any medium, provided the original work is properly cited.

\begin{abstract}
Background: Vibrio cholerae, the causative agent of cholera is known to cause outbreaks. It is crucial to identify these outbreaks and prevent transmission. Cases of acute diarrhea should be recognized promptly in order to prevent fatal outcome, with rationale use of antibiotics. This study was done to evaluate the burden of cholera cases and to determine their antibiotic sensitivity pattern.

Materials and methods: This was a retrospective study conducted in the Department of Microbiology in a tertiary care hospital in North India. The stool samples received in the laboratory were processed by standard microbiological techniques for identification of V. cholerae. Serological confirmation was done and antibiotic sensitivity pattern was detected by Kirby Bauer's disk diffusion method.

Results: V. cholerae was isolated in 28 out of 1567 samples. Serotyping confirmed $68 \%$ of the cases belonging to V. cholerae 01 and $32 \%$ were V. cholerae 0139 serotype. The organism showed sensitivity to tetracycline, gentamycin, Amikacin and Norfloxacin. Resistant drugs included ampicillin and trimethoprim-sulphamethoxazole. Monsoon season showed an increased prevalence of cases.

Conclusions: Our study revealed an increase in number of cases during the monsoon season, implying lack of sanitary conditions. V. cholerae 01 Ogawa serotype was the predominant strain. Rehydration therapy is the gold standard in the treatment of cholera, and cautious use of antibiotics should be encouraged, with emerging resistance seen for ampicillin and trimethoprim-sulphamethoxazole.
\end{abstract}

Keywords: Antimicrobial resistance, cholera, diarrhea, India, vibrio cholerae.

\section{Introduction}

Vibrio cholerae are defined as Gram-negative, asporogenous rods that are straight or have a single, rigid curve. They are motile; most have a single polar flagellum, when grown in liquid medium. Most produce oxidase and catalase, and ferment glucose without producing gas [1]. The genus Vibrio consists of species occurring in marine environment preferring salty, warm and alkaline conditions [2]. Recent studies have indicated that the human body environment activates some vibrio genes; therefore the species take a more virulent form in the human body. The cholera toxin is the most important virulence factor produced by Vibrio [2]. Cholera outbreaks have been frequently reported from one or the other part of this country especially during monsoon. However, occasional sporadic cases have also been reported, majorly due to lack of proper sanitation. It is the responsibility of the microbiology laboratory to define the beginning of cholera epidemic, monitor the changes in antimicrobial resistance during that epidemic and also confirm that the epidemic is over. More than 200 serogroup of Vibrio cholera have been identified; the two most important pathogenic forms belong to "classical" or El Tor biotype of serogroup 01 [3].

Since diarrhoeal diseases can have a fatal outcome, if not diagnosed and treated promptly, need to identify the incidence of cholera cases is required. Since antibiotics are given as secondary therapy along with rehydration therapy, there is a need to know the sensitivity pattern of the drugs used. Therefore this retrospective study was done in order to find out the cases of diarrhea caused by Vibrio and their antimicrobial susceptibility pattern. 


\section{Materials and methods}

This retrospective study was conducted over a period of one year from 1st June 2011 to 30th June, 2012 in the Department of Microbiology, Christian Medical College and Hospital, Ludhiana, India, a tertiary care hospital. Stool samples received in the laboratory was proceeded to confirm the causative organism. The diagnosis was suggested by the clinical picture and confirmed by stool culture. Macroscopic examination of stool samples was done. Hanging drop preparation was made and observed for darting motility. All the samples were inoculated on blood agar, MacConkey agar and alkaline peptone water. Plating was also done on thiosulphate citrate bile salt sucrose agar (TCBS) and deoxycholate citrate agar (DCA) using standard microbiological techniques [4]. Slide agglutination was performed with Vibrio cholera polyvalent 01, monovalent Ogawa and Inaba, and Vibrio cholera 0139 antisera. Antibiotic sensitivity was performed on Mueller Hinton agar plates by the Kirby Bauer's disk diffusion method using the following antibiotics: ampicillin (10ug), trimethoprim-sulphamethoxazole (25 ug), tetracycline (30ug), gentamicin (10ug), Amikacin (10ug) and Norfloxacin (10ug). The antibiotic discs used were obtained from Hi-Media Ltd, Mumbai, India. The zone of inhibition was measured using Clinical Laboratory Standard Institute guidelines (CLSI).

\section{Results}

Out of total 1567 stool specimens received in the laboratory during the study period, Vibrio cholerae were isolated in 28 stool samples, giving a positivity rate of $1.8 \%$. On serotyping, 68\% (19) of these positive samples were identified as V. cholera 01 serotype and $32 \%$ (9) were V. cholera 0139 serotype. Ninety two percentages (26) of cases were encountered during monsoon season.

The prevalence of resistance pattern of the isolated species during the study period is as shown in the Table 1 . V. cholerae $\mathrm{O} 1$ strains showed maximum sensitivity to tetracycline, gentamicin, Amikacin, Norfloxacin, whereas high degree of resistance was seen to ampicillin and trimethoprim-sulphamethoxazole. All the isolates of V. cholerae O139 were susceptible to ampicillin, tetracycline, gentamicin and Amikacin, while low level of resistance was observed to trimethoprim-sulphamethoxazole and Norfloxacin.

\section{Discussion}

Most of the studies done showed an increase in number of cases in the monsoon season, the most important factor being the lack of sanitation and impure water supply [5], [6]. Our study has also showed a high percentage of the 01 serotype being isolated. The Inaba strain also has the potential to cause an outbreak. It is one of the emerging causes of an outbreak being under studies [7].

The gold standard of treatment consists of oral and intravenous rehydration therapy. The use of antibiotics is of secondary importance. In our study, the isolates depicted highest susceptibility to gentamicin, Amikacin and tetracycline; and high level of resistance was observed for ampicillin and trimethoprim-sulphamethoxazole. These findings were in concurrence to other studies in which the isolates were highly susceptible to aminoglycosides and tetracycline's [8], [9].

Table: Antibiotic resistance profiles of Vibrio cholerae O1 and O139

\begin{tabular}{lll}
\hline & V.cholerae $01(\mathrm{n}=19)$ & V.cholerae $0139(\mathrm{n}=9)$ \\
\hline Ampicillin & $18(94.7)$ & 0 \\
Tetracycline & $1(5.3)$ & 0 \\
Trimethoprim-sulfamethoxazole & $18(94.7)$ & $2(22.2)$ \\
Norfloxacin & $1(5.3)$ & $1(11.1)$ \\
Gentamicin & 0 & 0 \\
Amikacin & 0 & 0 \\
\hline
\end{tabular}

Footnote: Figure in parentheses indicates percentage

Resistance to fluoroquinolones has been reported in studies from India [10], however most of the isolates were found to be susceptible to fluoroquinolones (Norfloxacin) in the present study. A number of multidrug resistance strains are also emerging in clinical isolates of V. cholera 01; serotype Ogawa, including resistance to trimethoprim-sulphamethoxazole and ampicillin [11]. Other studies have showed the current emerging toxigenic Inaba strains of Eltor biotype, than the earlier classical biotype [12]. Our study has shown more prevalence of the Ogawa strain.

Vibrio induces biochemical changes in the intestinal epithelium. Throughout the infective stage, the organism does not invade the intestinal epithelial cells. The toxin production leads to secretion of large quantities of water and electrolyte in the lumen of intestine, resulting in severe dehydration. The fatal outcome of disease can be prevented by rigorous maintenance of circulation by replacement of body fluids.

Prevention depends on sanitary measures to prevent fecal-oral transmission; hand-washing and proper preparation of food; chlorination of water supplies; and sewage treatment and disposal. Strict personal hygiene is vital and drinking 
water should come from a clean piped supply or be boiled. Flies must be denied access to food. Parenteral vaccination with a killed suspension of V. cholera provides some protection. Oral vaccines containing killed V. cholera and the B subunit of cholera toxin are available but are of limited efficacy.

In epidemics, public education and control of water sources and population movement are vital. Mass single-dose vaccination and treatment with tetracycline are valuable. Disinfection of discharges and soiled clothing, and scrupulous hand-washing by medical attendants reduce the danger of spread.

\section{Conclusion}

On the basis of our study it was revealed that a significant increase in cases of Vibrio infection occurred during monsoon season, with V. cholera 01 Ogawa as the predominant serotype. The results also suggest that this organism has begun to develop resistance against ampicillin and trimethoprim-sulphamethoxazole and therefore these drugs should not be used as first line therapy.

\section{References}

[1] Baumann, P., Furniss, A. and Lee, V. (1984.). In Bergey's Manual of Systematic Bacteriology (eds. N.R. Krieg and J.G. Holt) the Williams \& Wilkins Co., Baltimore/London.

[2] R Pearson, Microbiology with Diseases of Body System, 5th ed, 22; 796

[3] Hendriksen RS, Price LB, Schupp JM, et al. Population genetics of Vibrio cholerae from Nepal in 2010: evidence on the origin of the Haitian outbreak. MBio. 2011 Sep 1; 2(4):e00157-11. doi: 10.1128/mBio.00157-11

[4] Barron EJ, Finegold SM. In: Bailey \& Scott's diagnostic microbiology, Missouri, USA: CV Mosby Company; 1990. p. $431-5$.

[5] Dalsgaard, A, Albert, MJ, Taylor DN. et al. Characterization of Vibrio cholera non 01 serogroups obtained from an outbreak of diarrhoea in Lima, Peru. J Clin Microbiol. 1995 Oct; 33(10):2715- 22.

[6] Sack RB, Siddique AK, Longini IM, Jr., Nizam A, Yunus M, Islam MS, et al. A 4-year study of the epidemiology of Vibrio cholerae in four rural areas of Bangladesh. J Infect Dis. 2003 Jan 1; 187(1):96-101.

[7] Taneja N, Biswal M, Tarai B, Sharma M. Emergence of Vibrio cholerae O1 Biotype El Tor serotype Inaba in north India. Jpn J Infect Dis. 2005 Aug; 58(4):238-40.

[8] Taneja N, Kaur J, Sharma K, Singh M, Kalra JK, Sharma NM, et al. A recent outbreak of cholera due to Vibrio cholerae O1 Ogawa in \& around Chandigarh, North India. Indian J Med Res. 2003 Jun; 117:243-6.

[9] Sharma NC, Mandal PK, Dhillon R, Jain M. Changing profile of Vibrio cholerae O1, O139 in Delhi \& its periphery (2003-2005). Indian J Med Res. 2007 May; 125(5):633-40.

[10] Mukhopadhyay AK, Basu I, Bhattacharya SK, Bhattacharya MK, Nair GB. Emergence of fluoroquinolone resistance in strains of Vibrio cholerae isolated from hospitalized patients with acute diarrhea in Calcutta, India. Antimicrob Agents Chemother. 1998 Jan; $42(1): 206-7$.

[11] Kutar BM, Rajpara N, Upadhyay H, Ramamurthy T, Bhardwaj AK. Clinical isolates of Vibrio cholerae O1 El Tor Ogawa of 2009 from Kolkata, India: preponderance of SXT element and presence of Haitian ctxB variant. PLoS One. 2013 ; 8(2):e56477. doi:10.1371/journal.pone.0056477.

[12] Garg P, Nandy RK, Chaudhury P, Chowdhury NR, De K, Ramamurthy T, et al. Emergence of Vibrio cholerae O1 biotype El Tor serotype Inaba from the prevailing O1 Ogawa serotype strains in India. J Clin Microbiol. 2000 Nov; 38(11):4249-53. 